\title{
SENSOR NETWORK COVERAGE RESTORATION
}

\author{
Nitin Kumar, Dimitrios Gunopulos \& Vana Kalogeraki \\ Department of Computer Science \& Engineering \\ University of California \\ Riverside, California 92521 \\ \{nkumar, dg, vana\}@cs.ucr.edu
}

\begin{abstract}
Wireless sensor networks are emerging as a new computational platform consisting of small, low-power and inexpensive nodes used in a broad set of application areas including environmental monitoring, habitat monitoring and disaster recovery. Typically sensor nodes are deployed over a geographical area for the purpose of detecting, tracking and monitoring events of interest. Since sensor nodes are deployed in a large land region, the objective is to achieve complete coverage of the region, that is, every location in the region lies in the observation field of at least one sensor node. However the initial placement of sensors may not achieve this goal for various reasons: the number of original sensors may have been too low, the original placement may have been random (for example, sensors deployed from the air) leaving parts of the region uncovered, or, some of the sensors have malfunctioned, leaving coverage holes.

In this paper we consider the coverage restoration problem in sensor networks. The goal is to find a minimal set of new sensors, and their locations, such that when they are added to an existing sensor field we can achieve complete coverage of the region under surveillance. The technique we propose is distributed, and minimizes the communication costs. The key idea of our technique is to use an efficient and yet very accurate representation of the uncovered area that uses techniques from discrepancy theory. By representing the uncovered area as a set of points, we can use efficient and simple algorithms for finding small sets of sensors to cover the uncovered areas. We partition the sensor network into cells, and run these algorithms locally. We also present an extensive experimental evaluation to validate our approach.
\end{abstract}

Keywords: Sensor Networks, Coverage, Restoration

\section{Introduction}

Sensor networks are expected to be deployed over a geographical area for the purpose of detecting, tracking and monitoring events of interest. Reports produced upon the observation of specific events are typically 
processed locally at the sensor nodes and transmitted over multiple hops to a centralized sink in order to reach an operations center or to be analyzed further. These have found a growing number of applications including environmental monitoring, habitat monitoring and disaster recovery. Recently, a lot of research has been conducted in sensor networks related to the physical and media access layers, routing and transport protocols [3][5][17] and localization and positional applications of sensor networks [1][10].

In this paper we study the coverage restoration problem in sensor networks. The fundamental question is "Given a two-dimensional area, a piece of land for example, and an initial set of sensors, how can we determine the number of sensor nodes required to completely cover the region". Essentially, the coverage restoration problem reflects how well an area is monitored by sensors. In abstract terms, our approach determines uncovered area in the sensor network field and proposes the deployment of nodes to completely cover the area. Our mechanism consists of two steps: (a) estimating the regions uncovered by sensors and (b) identifying the minimum number and location of sensors required to cover this region. The key idea of our technique is to make an efficient and yet very accurate representation of the uncovered area that uses techniques from discrepancy theory. By representing the uncovered area as a set of points, we can use efficient and simple algorithms for finding small sets of sensors to cover the uncovered areas. We partition the sensor network into cells, and run these algorithms locally. We formulate this problem as a disk covering problem, where the goal is to cover a set of points on the plane by a set of disks. This problem is known to be NP-complete [12]. However, there exist various approximate solutions that run in polynomial time and have a bounded error ratio [9]. We will use one of such proposed algorithm for our experimental purpose.

The coverage problem has many applications in various practical problems. As an example, consider the million acres of land areas, which get destroyed due to forest fires every year. If temperature-sensing nodes could be deployed to fully cover these sensitive regions, early warnings from sensors can help preventing such infernos. Another example could be monitoring of plants in a field. To monitor the growth of a group of plants arrayed in a field, we need a mechanism to place sensor nodes in such a way that every plant gets covered by at least one sensor node.

\section{Our Contribution}

We propose a solution for the coverage restoration problem in wireless sensor area networks. Our technique consists of the following novel 
components: (a) an efficient and very accurate method for representing an uncovered sensor area using techniques from discrepancy theory, and (b) a distributed mechanism for identifying a small number and location of nodes required to cover the sensor area.

The remainder of this paper is organized as follows: Section 1 discusses previous research related to the coverage problem in sensor networks. Section 2 describes the problem statement and Section 3 describes our approach to the solution. Section 4 provides a detailed performance evaluation and comparison results. We conclude the paper in Section 5.

\section{Related Work}

The coverage problem of wireless sensor networks has been studied recently. The authors in [4], formulate this problem as a decision problem and determine whether every point in the service area of the sensor network is covered by at least $\mathrm{k}$ pre-defined sensors. In [14], an algorithm is proposed for coverage problem of a wireless ad-hoc sensor network. They have assumed a centralized control server, where nodes are connected using a gateway. The problem of efficient coverage of an area with base stations for wireless networks looks like a similar problem. However, there are significant differences that do not allow a straightforward implementation of base station solutions in sensor networks. The number of sensor nodes is significantly higher than the number of base stations. Also a base station has a broader coverage capacity compared to the tiny sensor nodes. The coverage problem has also been discussed in [15][18]. The authors in [15] solve the problem of best-coverage path between any pair of sensor nodes using Delaunay triangulation and the Vornoi diagram. They assume a centralized control server, where nodes are connected using gateway. Their approach may not be feasible for a large network where nodes are scattered over multiple hops. In [18], the authors proposed a distributed algorithm using the relative neighborhood graph. They extended the best coverage problem to find out a path with a) best coverage distance while the total energy consumed by this path is minimized among all optimum best coverage-paths, and b) best coverage distance with the total length of edges of this path being no more than 2.5 times the shortest best coverage path. A problem very similar to ours was tackled in [14]. They propose a method for area coverage with a heuristic that organizes the available sensor nodes into mutually exclusive sets where the members of each of those sets completely cover the monitored area. All these methods assume random deployment of sensor nodes which may or may-not provide a full coverage. Yan et al. [19]introduce an adaptable sensing coverage 
mechanism for differentiated surveillance in sensor networks. They propose a scheme to full sensing coverage as well as a degree of coverage up to the limit imposed by the density of nodes available. Santosh et al. [13]proposed a mechanism to determine the appropriate number of sensors to deploy that achieves $\mathrm{k}$ coverage of protected regions and in addition improves the lifetime of individual sensors. They considered three kinds of deployments for a sensor network on a unit square - an $\sqrt{n} \times \sqrt{n}$ grid, random uniform sensor distribution(for all $\mathrm{n}$ points), and Poisson distribution(with density $\mathrm{n}$ ). The authors in [8]presented an analytical model under probabilistic coverage to investigate the objecttracking quality with respect to various network conditions and sensor scheduling schemes.

\section{Problem Statement for Network Coverage Restoration}

We consider the case where we already have a set of sensors installed in a 2-dimensional area $A$, but these sensors do not completely cover the field. A location in area $A$ is said to be covered, if it lies in the sensing range of at least one sensor node. The sensing range of a sensor node is defined by radius $r$, i.e. it can monitor any object that is within a distance of $r$ from it.

The problem we consider here is how to efficiently identify the areas that are not covered (see figure 1) and how to restore the desired coverage levels with minimal cost (that is, minimizing the number of additional sensors). We make an assumption that the sensor nodes are either GPS enabled or they are capable of finding out and reporting their respective positions to the leader node.

\section{Solution Approach}

In this section, we describe a distributed covering algorithm, which runs in the sensor networks using the computational resources of nodes. The given region is partitioned into local regions called cells in such a way that the sensors in each cell are able to communicate with each other efficiently to form a network between all the sensors in the cell. We propose a hierarchical network organization in which a randomly elected leader will represent each local cell. We assume that each sensor knows its location, and that the leader at least knows the geographical boundaries of its cell. Each leader is responsible for sending a description of the uncovered regions in its cell to the base station.

We make the assumption that there is at least one sensor in each cell, to act as a leader. This is without loss of generality, because if an en- 
tire cell is empty, we can use a regular positioning of sensors to cover it. The selection of leader can be done using an energy-efficient scheme that periodically assigns leaderships to different sensors in each cell. We propose a random selection of leaders and a rotation mechanism for leadership selection so that the energy dissipation experienced by the leader in communicating with sensors gets spread across all nodes in the cell. The leader selection algorithm is performed periodically in each cell. Several approaches have been proposed for leader selection procedures [11]. Liu et al. [8] implemented a geographically based group management scheme that uses time-stamped messages to solve the contentions and elect a single leader in a region. Another approach introduced in [17] uses randomization to distribute the energy load evenly among the nodes in the network. Each leader independently runs a centralized algorithm in its cell that performs the following two steps:

1 Estimates the uncovered region.

2 Identify a small number of sensors and their respective location that are needed for complete coverage.

We now describe each of the steps in details:

\subsection{Estimating the Uncovered Region}

For a given partially covered cell, the leader must first estimate all the uncovered areas. We propose to do this by finding a simple description of the uncovered area. For simplicity, instead of producing an actual description of the uncovered area, we produce an implicit description by finding a set of points that are not covered. This has the following advantages:

1 The algorithm is simple and can be executed locally. We have to find for each point if it is covered or not, which can be done easily locally.

2 The description of the uncovered area is also very simple: It consists of a set of discrete points.

However, the accuracy of the algorithm depends on how well the chosen set of points approximate the area. This problem (the problem of approximating a continuous measure such as the area with a discrete measure such as a set of points) has been studied extensively in the area of Monte Carlo and Quasi-Monte Carlo integration. It has been shown that there exist sequences of 2-dimensional points that approximate the area much better than a random set of points of equal cardinality. 


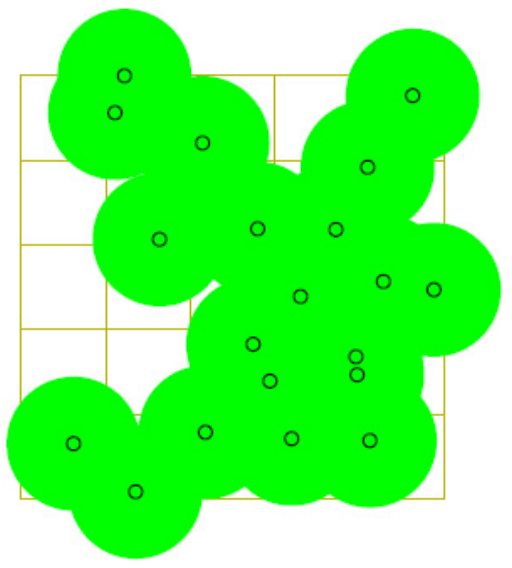

Figure 1: Initial 20 sensor nodes with their coverage area (radius $=0.79)$

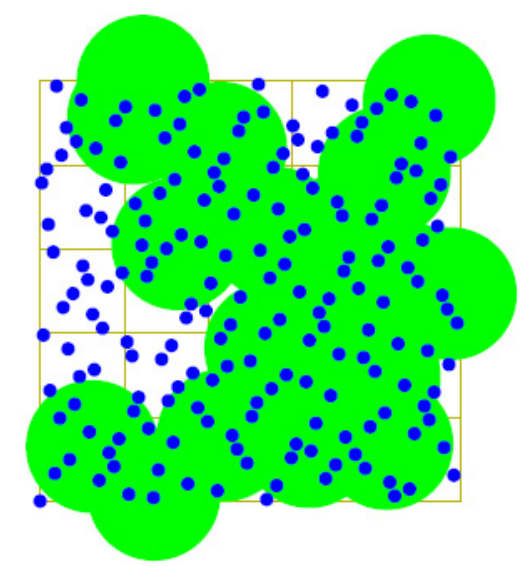

Figure 2: Halton distribution of 187 points to estimate the uncovered regions.

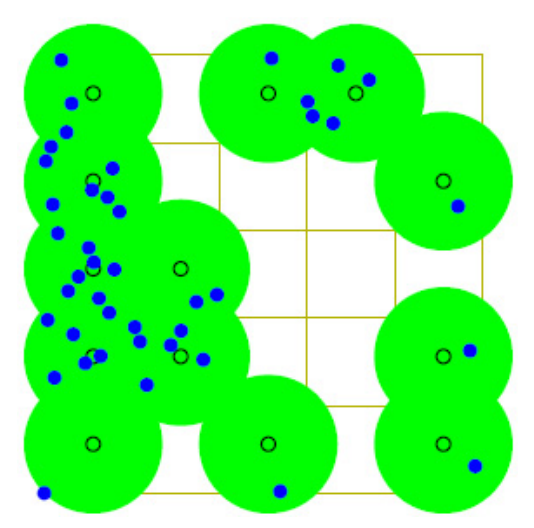

Figure 3: 13 extra sensors covering 42 uncovered points

Such point sets are characterized by low-discrepancy [16][6]. For choosing a set of points to approximate the uncovered region, we propose to use the Halton and Hammersely generator which generates low discrepancy points for dimension $d$, of the order of $\mathrm{O}\left(\frac{\log ^{d} N}{N}\right)$ and $\mathrm{O}\left(\frac{\log ^{d-1} N}{N}\right)$ respectively, when a random set of points would have $\mathrm{O}\left(\sqrt{\frac{\log \log N}{N}}\right)$ discrepancy. The leader eliminates all those points from the point set in its cell, which lie in the coverage area of the previously placed sensor nodes (see figure 2) so that the remaining points give us roughly a close estimation of all the uncovered regions in that cell. 


\subsection{Calculating the Number of Sensors Needed to Completely Cover the Uncovered Regions}

Once the leader find an estimate of the uncovered regions in the form of discrete points in their respective cells, the problem gets reduced to a disk-covering problem, the goal being to cover uncovered points with a minimum numbers of disks (extra sensor nodes, in this case). We apply a covering algorithm to achieve this task. A covering algorithm places disks on the plane, according to distribution of the points, in such a way that each disk covers at least one point and each point is covered by at least a disk [2] (see figure 3). We next discuss the covering algorithm in the section 3.2.1. The covering algorithm gives us the location of the extra sensor nodes required to fully cover the uncovered regions.

3.2.1 Covering Algorithm. We have used a combination of the polynomial scheme of applying shifting technique proposed by Hochbaum et al. [7] and the grid strategy of covering points by placing disks only at the vertices of a grid proposed by Franceschetti et al. [9]. The approach works by partitioning the area into vertical strips, and covering the points in each strip. To cover $n$ given points by disks of diameter $D$ in a 2-dimensional area, we apply the shifting strategy in two phases. Considering the shift parameter as $t$, we cut the plane into vertical strips of width $t \times D$ (i.e. taking a group of $t$ consecutive strips of width $D$ each). This partitioning of plane into strips of width $t \times D$ can be done in $t$ different ways $\left(S_{1}, S_{2}, \ldots, S_{t}\right)$, where we derive each way from the previous one by shifting it to the right over distance $D$. In order to cover points on each strips, we apply the same shifting strategy in the other dimension, i.e. the considered strip is cut into squares of side $t \times D$. Then, we apply the grid strategy to find out the optimal covering of points in such a square. As we know that we can cover a square of side $t \times D$ compactly with $(\lceil t \sqrt{2}\rceil)^{2}$ disks of diameter $D$. By checking all possible arrangement of $(\lceil t \sqrt{2}\rceil)^{2}$ disks, an optimal covering within the square can be discovered. We sum up together the best solutions in each square to completely cover the considered partition. Finally we compute the solution for each of the partitions $S_{1}, S_{2}, \ldots, S_{t}$ and select the partition which gives the best solution.

3.2.2 Applying the Covering Algorithm. In our approach, the covering algorithm runs independently in each cell using the network partitioning discussed in section 3. Each cell gets covered independently providing a global solution in a distributed fashion. The leader decides where to add extra sensors in its area, without any need to transmit all 


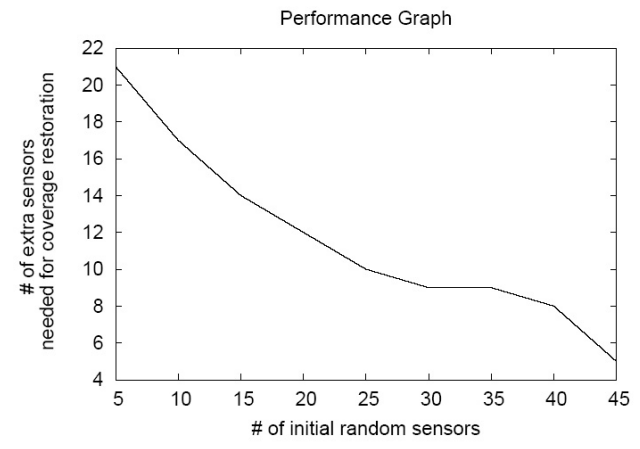

Figure 4: Extra sensors required for a given initial randomly distributed sensors on a grid of $5 \times 5$.

the data back to the base station. The decisions can be retrieved from leaders and the required sensors can be deployed by the base station.

An advantage of our distributed approach is that we minimize the communication cost and the energy consumption, because we keep all sensor to sensor communication contained inside the cell. A disadvantage is that each leader has to have enough computational resources that it can run the Halton algorithm to compute the set of low discrepancy points and then run the coverage algorithm. To alleviate these problems, the region that each leader is responsible for, is kept relatively small, so that we need a small point set of low discrepancy, and so the running time of the covering algorithm is also small.

As an example, we present a simulation result of the algorithm run on a square grid of $5 \times 5$ cells. We assume that the coverage radius of each sensor is 0.79 . Figure 1 shows the initial random distribution of 20 sensor nodes with their coverage area. Each sensor node covers a circular area of radii 0.79 , shown by colored circles. Figure 2 shows the Halton low discrepancy distribution of points. This distribution contains a large density of Halton points scattered all around the region in order to cover the entire area. The number of such points is 187 , out of which, we eliminate all those points which lies in the coverage area of the initially placed sensor nodes. The remaining points represent the uncovered area. Figure 3 shows 42 uncovered points covered by 13 extra sensors.

\section{Experimental Results}

In this section, we test our proposed approach with a comprehensive set of experiments. We conducted experiments to show the effect of varying the number of initial sensor nodes on the number of extra sensors required to completely cover the region. As shown in Figure 4, 


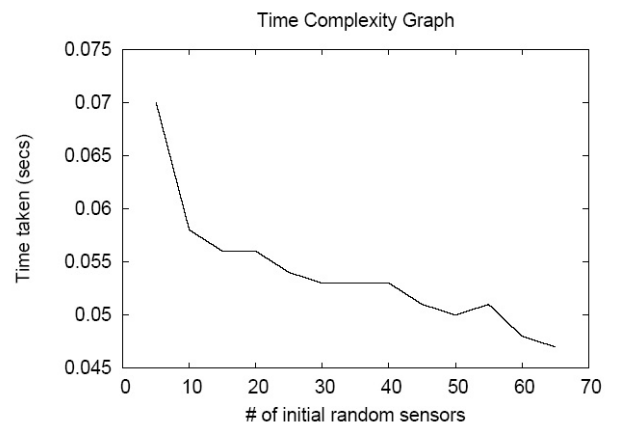

Figure 5: Time complexity vs the number of initial random sensors

the number of extra sensor nodes needed to completely cover the region decreases as we increase the number of initial sensor nodes. In another experiment we evaluated the accuracy of our algorithm in terms of the percentage of total area covered. By varying the initial number of sensor nodes, we applied the algorithm to obtain a good covering of the uncovered regions. We observe that, on average, we could cover $99.9 \%$ of the uncovered regions in a single pass of the algorithm, with a standard deviation of error 0.0013 and worst-case accuracy of $99.88 \%$.

We also conducted an experiment to observe the running time of our algorithm depending on the number of initial sensor nodes on a $10 \times 10$ grid. The graph in Figure 5 shows that as the initial number of sensor nodes increases, the time required to determine the uncovered regions and covering them with the optimal number of sensor nodes decreases. The algorithm runs faster with the increase in number of initial sensor nodes, because it decreases the uncovered area and hence the number of uncovered points by the program also gets decreased.

\section{Conclusions and Future Work}

We have presented a method to calculate the number of extra sensor nodes required to completely cover a given region. Our approach is unique in that it can be applied globally or by diving the region into various local sub-areas and applying the algorithm in each of the local areas. We have demonstrated through simulations that our technique is highly effective in achieving a good coverage of a given sensor network area. 


\section{References}

[1] P. Bahl and V. N. Padmanabhan. Radar: An in-building rf-based user location and tracking system. IEEE INFOCOM, Tel-Aviv, Israel March 2000.

[2] L. Booth, J. Bruck, M. Franceschetti \& R. Meester. Covering algo, continuum percolation \& geometry of wireless network. Annals, Applied Probability, 2003.

[3] D. Braginsky, D. Estrin. Rumor routing Algorithm for sensor networks. International Conf. on Distributed Computing Systems,ICDCS, Austria July 2002.

[4] Y. Tseng, C. Huang. Coverage problem in wireless sensor network. ACM International Conference WSNA, San Diego, CA, USA September 2003.

[5] S. Shenker, D. Ganesan, R. Govindan \& D. Estrin. Highly resilient, energy efficient multipath routing in wireless sensor networks. ACM SIGMOBILE Mobile Computing and Communication, Rome, Italy July 2001.

[6] J.Hammersley,Handscomb. D.C.Monte Carlo Method. Methuen, London 1964.

[7] D. S. Hochbaum and W. Maass. Approximation schemes for covering and packing problems in IP and VLSI. pages 130-136. Journal of ACM 1985.

[8] J.J. Liu, J. Liu, J. Reich, P. Cheung, and F. Zhao. Distributed group management for track initiation and maintenance in target localization applications. 2nd International Workshop on IPSN . pp 113-128, 2003.

[9] M. Cook, M. Franceschetti and J. Bruck. A geometric theorem for approximate disk covering algorithms. TR, California Inst. of Technology 2001.

[10] J. Heidemann, N. Bulusu and D. Estrin. Gps-less low cost outdoor localization for very small devices. IEEE Personal Comm. Magazine 7(5):28-34, Oct 2000.

[11] J. Welch, N. Malpani and N. Vaidya. Leader election algorithms for mobile ad hoc networks. ACM DIAL M, Massachusetts 2000.

[12] M. Paterson, R. Fowler and S. Tanimoto. Optimal packing and covering in the plane are NP complete. Information Processing Letters, 12(3):133-137, 1981.

[13] Ten Hwang Lai, Santosh Kumar and Jozsef Balogh. On k-coverage in a mostly sleeping sensor network. ACM Mobicom, Philadelphia, PA, 2004.

[14] Miodrag Potkonjak and Sasa Slijepcevic. Power efficient organization of wireless sensor networks. IEEE ICC, Helsinki, Finland, pp 472-476, June 2001

[15] M. Potkonjak, S. Meguerdichian, F. Koushanfar and Mani B. Srivastava. Coverage problems in wireless sensor networks. IEEE INFOCOM, Alaska, 2001.

[16] H. Wozniakowski. Average case complexity of multivariate integration. volume 24, pages 185-194. Bull. Amer. Math. Society, 1991.

[17] A.Chandrakasan, W.R.Heinzelman and H.Balakrishnan. Energy-efficient communication protocols for wireless microsensor networks. In proceedings of Hawaii International Conference on system Sciences, Hawaii, January, 2000.

[18] P.Wan, X.Li \& O.Frieder, Coverage in wireless sensor networks, ICC, 2002.

[19] T. Yan, T. He, and J. Stankovic. Differentiated surveillance for sensor networks. ACM Sensys,Los Angeles, CA, Nov 2003. 